\title{
Untying links through anti-parity-time-symmetric coupling
}

\author{
H. C. Wu, X. M. Yang, L. Jin $\oplus^{*}$ and Z. Song ${ }^{\dagger}$ \\ School of Physics, Nankai University, Tianjin 300071, China
}

(Received 9 July 2020; revised 17 September 2020; accepted 21 September 2020; published 5 October 2020)

\begin{abstract}
We reveal how the vector field links are untied under the influence of anti-parity-time-symmetric couplings in a dissipative sublattice-symmetric topological photonic crystal lattice. The topology of the quasi-one-dimensional two-band system is encoded in the geometric topology of the vector fields associated with the Bloch Hamiltonian. The linked vector fields reflect the topology of the nontrivial phase. The topological phase transition occurs concomitantly with the untying of the vector field link at the exceptional points. Counterintuitively, more dissipation constructively creates a nontrivial topology. The linking number predicts the number of topological photonic zero modes.
\end{abstract}

DOI: 10.1103/PhysRevB.102.161101

\section{INTRODUCTION}

The robust topological structures of monopole, skyrmion, and vortex have attracted much research interest in optics, quantum physics, and condensed matter physics. The zerointensity lines of light field in the form of isolated knotted nodal lines are observed as optical vortices in experiments [1]. The creation, observation, and investigation on the static and dynamical properties of quantum knots in Bose-Einstein condensates are reported [2]. Besides, topological phases are extremely stable [3-11]. In topological systems, zero-energy Fermi surfaces can form knotted or linked nodal lines [12-18]; alternatively, the fictitious magnetic field of a topological system with topological defects, associated with vortex or antivortex textures, reflects nontrivial topology $[19,20]$. In this Rapid Communication, a nontrivial topology is extracted from the spin polarization, which is associated with a two-band topological system and defines a vector field. The vector field exhibits a link or knot topology in the topologically nontrivial phase. We visualize the vortex links, present alterations of the vector field topology under the influence of anti-paritytime-symmetric (anti- $\mathcal{P} \mathcal{T}$-symmetric) couplings, and reveal the untying of vector field links associated with a topological phase transition at the exceptional point (EP) of the nonHermitian topological system. Counterintuitively, we demonstrate that more dissipation constructively creates nontrivial topology.

\section{DISSIPATIVE PHOTONIC CRYSTAL LATTICE}

Photonic crystal is an excellent platform for the study of topological physics [6]. The non-Hermitian quasicrystal

\footnotetext{
*jinliang@nankai.edu.cn

†songtc@nankai.edu.cn
}

Published by the American Physical Society under the terms of the Creative Commons Attribution 4.0 International license. Further distribution of this work must maintain attribution to the author(s) and the published article's title, journal citation, and DOI.
[21,22], high-order topological phase [23-27], robust edge state [28-36], and topological lasing [37-43] in photonic crystals were discovered. Here, we consider a photonic crystal lattice as schematically illustrated in Fig. 1(a), which is implementable in many experimental platforms including the hybrid silicon microcavities [36-38], the optical waveguides [32,44,45], and the dielectric microwave resonators $[29,39]$. The auxiliary resonators in white and gray are evanescently coupled to the primary resonators in red and blue. The primary resonators have an identical resonant frequency $\omega_{c}$. The auxiliary resonators induce the effective couplings between primary resonators. The blue, red, and white resonators are passive; the gray resonators have dissipation. The dissipation rate is much larger than the coupling strength between the gray auxiliary resonators and the colored primary resonators. The large dissipation enables the adiabatical elimination of the auxiliary resonator light field and results in an anti- $\mathcal{P} \mathcal{T}$-symmetric reciprocal coupling $-i \gamma$ between two adjacent primary resonators [46]; under the on-resonance condition, the coupling strength $\gamma$ equals to the product of two evanescent couplings between the gray auxiliary resonator and its two adjacent primary resonators divided by the dissipation rate of the gray auxiliary resonator. The other two effective coupling strengths induced by the white auxiliary resonators are denoted as $J$ and $\kappa$.

In coupled mode theory [47], the equations of motion for the coupled resonator array are

$$
\begin{aligned}
& i \frac{d}{d t} \psi_{A, j}=\omega_{c} \psi_{A, j}-i \gamma \psi_{B, j}+J \psi_{B, j-1}+\kappa \psi_{B, j-2}, \\
& i \frac{d}{d t} \psi_{B, j}=\omega_{c} \psi_{B, j}-i \gamma \psi_{A, j}+J \psi_{A, j+1}+\kappa \psi_{A, j+2},
\end{aligned}
$$

where $\psi_{A, j}$ and $\psi_{B, j}$ are the wave-function amplitudes for the corresponding sites $j$ of the sublattices $A$ and $B$. The schematic of the quasi-one-dimensional (quasi-1D) bipartite lattice is shown in Fig. 1(b).

Applying the Fourier transformation, the equations of motion for the eigenmode with momentum $k$ 


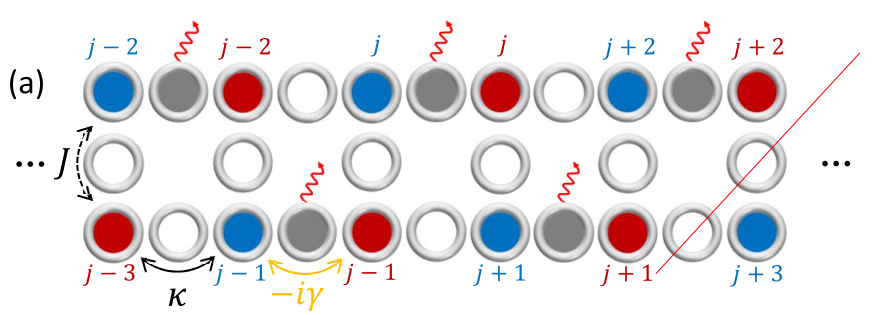

(b)

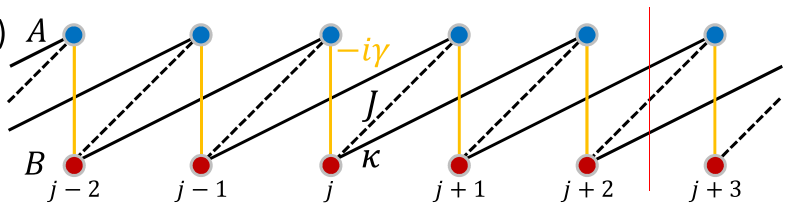

FIG. 1. (a) Quasi-1D coupled resonator array. The primary resonators (in blue and red) are effectively coupled by the auxiliary resonators (in gray and white). (b) Schematic of (a). The open boundary of (b) corresponds to the sideling cut of (a), indicated by the solid red lines.

reduce to

$$
\begin{gathered}
i \frac{d}{d t} \psi_{A, k}=\omega_{c} \psi_{A, k}+\left(-i \gamma+J e^{-i k}+\kappa e^{-2 i k}\right) \psi_{B, k}, \\
i \frac{d}{d t} \psi_{B, k}=\omega_{c} \psi_{B, k}+\left(-i \gamma+J e^{i k}+\kappa e^{2 i k}\right) \psi_{A, k} .
\end{gathered}
$$

After the common resonant frequency $\omega_{c}$ is removed for both sublattices, the two-band system is described by the Bloch Hamiltonian $h_{k}=(\vec{d}-i \vec{\Gamma}) \cdot \vec{\sigma}$, where $\vec{\sigma}=$ $\left(\sigma_{x}, \sigma_{y}, \sigma_{z}\right)$ is the Pauli matrix for spin- $1 / 2$ and $\vec{d}-i \vec{\Gamma}$ is the fictitious magnetic field including the real part $\vec{d}=$ $(J \cos k+\kappa \cos (2 k), J \sin k+\kappa \sin (2 k), 0)$ and the imaginary part $\vec{\Gamma}=(\gamma, 0,0)[48,49]$. Notably, the quasi-1D lattice and its Bloch Hamiltonian $h_{k}$ are not anti- $\mathcal{P} \mathcal{T}$-symmetric. The two energy bands are

$$
\varepsilon_{k}^{ \pm}= \pm\left[\left(d_{x}-i \gamma\right)^{2}+d_{y}^{2}\right]^{1 / 2}
$$

The corresponding eigenmodes are

$$
\left|\psi_{k}^{ \pm}\right\rangle=\left[1,\left(d_{x}-i \gamma+i d_{y}\right) / \varepsilon_{k}^{ \pm}\right]^{T} / \mathcal{N},
$$

which are normalized to unity $\left\langle\psi_{k}^{ \pm} \mid \psi_{k}^{ \pm}\right\rangle=1$. The average values of Pauli matrices under the eigenmode yield a physical observable of spin polarization $[19,20]$ and define a momentum dependent unity $3 \mathrm{D}$ vector field $\mathbf{F}_{ \pm}(k)=$ $\left(F_{ \pm, x}(k), F_{ \pm, y}(k), F_{ \pm, z}(k)\right)$. All of the three components $F_{ \pm, x, y, z}(k)=\left\langle\psi_{k}^{ \pm}\left|\sigma_{x, y, z}\right| \psi_{k}^{ \pm}\right\rangle$are gauge independent periodic functions of the momentum $k$ and can be expressed as

$$
\mathbf{F}_{ \pm}(k)=\left(\sin \theta_{ \pm} \cos \phi_{ \pm}, \sin \theta_{ \pm} \sin \phi_{ \pm}, \cos \theta_{ \pm}\right) .
$$

The information of the vector field is encoded in $\left(\theta_{ \pm}, \phi_{ \pm}\right)$. In knot theory, 1D topology describes 1D closed curves in 3D space [50].

\section{LINKING TOPOLOGY}

Figure 2 shows the vector fields $\mathbf{F}_{+}(k)$ and $\mathbf{F}_{-}(k)$ in different topological phases; they are mapped onto a torus as two closed curves, and their interrelation provides a visualization of the band topology of $h_{k}$ : the linked vector field curves represent the nontrivial topology. In Fig. 2, the torus has a major radius of $R_{0}$, which is the distance from the center of the tube to the center of the torus. $r_{0}$ is the minor radius of the tube. $k$ is taken as the toroidal direction, and $\mathbf{R}_{ \pm}(k)=\left(R_{0}+r_{ \pm} \cos \phi_{ \pm}\right) \vec{e}_{r}+r_{ \pm} \sin \phi_{ \pm} \vec{e}_{z} ; r_{ \pm}=r_{0} \sin \theta_{ \pm}$is selected to ensure that $\mathbf{R}_{ \pm}(k)$ forms a closed curve. The curve detaches from the surface of the torus when $\left|r_{ \pm}\right| \neq r_{0}$. The braiding of the two vector field curves is characterized by the linking number

$$
L=\frac{1}{4 \pi} \oint_{k} \oint_{k^{\prime}} \frac{\mathbf{R}_{+}(k)-\mathbf{R}_{-}\left(k^{\prime}\right)}{\left|\mathbf{R}_{+}(k)-\mathbf{R}_{-}\left(k^{\prime}\right)\right|^{3}} \cdot\left[d \mathbf{R}_{+}(k) \times d \mathbf{R}_{-}\left(k^{\prime}\right)\right] .
$$

Different links are topologically inequivalent and cannot be continuously deformed into one another without a topological phase transition. (a)
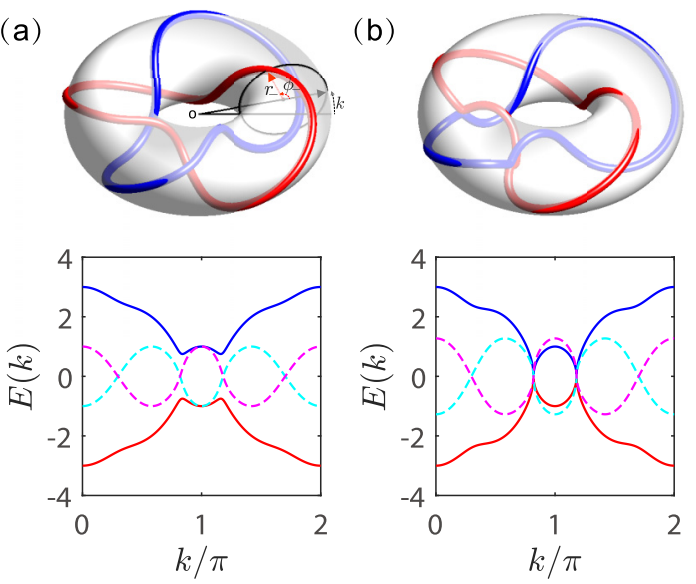

(b)

(c)
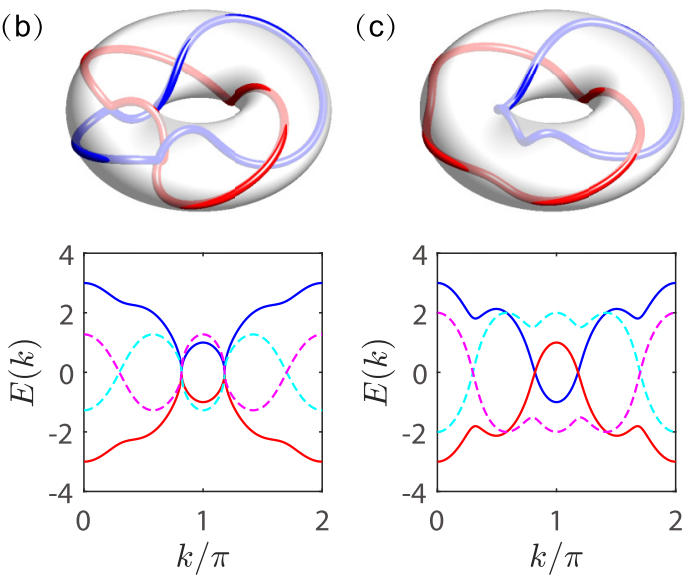

(d)

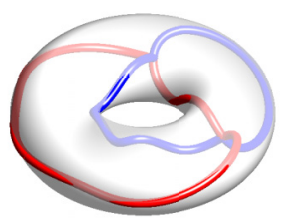

(e)
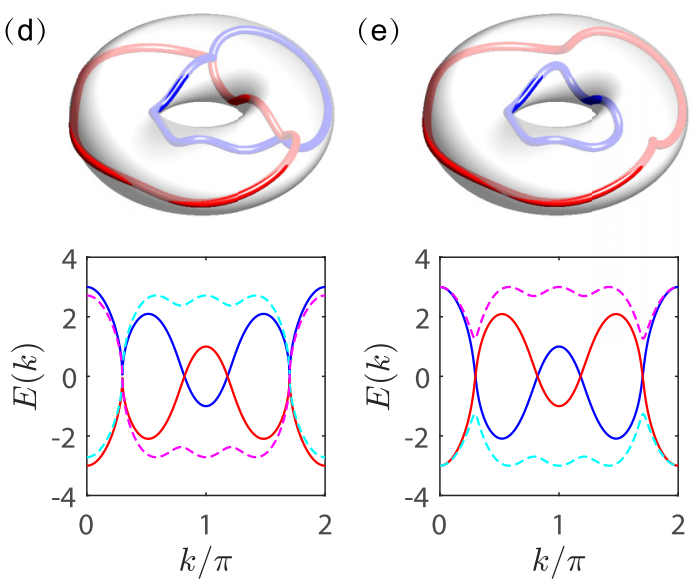

FIG. 2. Vector field curves $\mathbf{F}_{ \pm}(k)$ and energy bands for distinct topological phases. (a) $\gamma=1$, (b) $\gamma=1.274$, (c) $\gamma=2$, (d) $\gamma=2.715$, and (e) $\gamma=3$. The linking numbers are $L=-2,-1$, and 0 for (a), (c), and (e); (b) and (d) are the gapless phases. Other parameters are $J=1$ and $\kappa=2$. The energy bands are fully complex except at the crossing points of their imaginary parts. 
In the Hermitian case $(\gamma=0)$, the band energy is real and $F_{ \pm, z}(k)=0$; subsequently, $\theta_{ \pm}= \pm \pi / 2$ and $\left|r_{ \pm}(k)\right|=r_{0}$. Both vector field curves are located on the surface of the torus. The orthogonality of eigenmodes in the Hermitian system yields $r_{ \pm}(k)= \pm r_{0}$. In the presence of non-Hermiticity $(\gamma \neq 0)$, the energy band is complex, and $\theta_{ \pm} \neq \pm \pi / 2$. The minor radius for the vector field curve is $\left|r_{ \pm}(k)\right| \neq r_{0}$, because of the nonorthogonality of eigenmodes in the non-Hermitian system, and the two vector field curves detach from the torus surface.

In Fig. 2(a), the two vector field curves form a Solomon's knot with the linking number $L=-2$. As non-Hermiticity increases, two independent vector field curves meet at the EPs [51-53] and form a network [Fig. 2(b)]. The EPs are the nodes of the network with $\mathbf{F}_{+}(k)=\mathbf{F}_{-}(k)$ as a consequence of eigenmode coalescence. As non-Hermiticity increases, the curves reform as two independent closed loops; the vector field link changes through the reconnection associated with the EPs, and the linking number of the vector field curves is reduced by one, forming a Hopf link with linking number $L=-1$ [Fig. 2(c)]. This exhibits the untying of the link topology through the anti- $\mathcal{P} \mathcal{T}$-symmetric coupling. After another EP occurs at even greater anti- $\mathcal{P} \mathcal{T}$-symmetric coupling strength [Fig. 2(d)], the two vector field curves are unlinked [Fig. 2(e)]. The linking number associated with this phase is $L=0$ and the band is topologically trivial. Notably, the anti$\mathcal{P} \mathcal{T}$-symmetric coupling induces the topological phase transition at the EP, which dramatically differs from the topological phase transitions in other non-Hermitian systems with $\mathcal{P} \mathcal{T}$ symmetric gain and loss [28-34], as well as in non-Hermitian systems with asymmetric coupling strengths [54-59], where topological phase transition is irrelevant to the EP. Counterintuitively, increasing the dissipation can create nontrivial topology instead of behaving destructively: more dissipation reduces the strength of the anti- $\mathcal{P} \mathcal{T}$-symmetric coupling, tying vector field link and increasing the linking number.

The linking topology is closely related to the vortex associated with the topological defects in the vector field. For the photonic crystal lattice under consideration, the real part of the fictitious magnetic field satisfies $d_{x}(k)=$ $d_{x}(-k), d_{y}(k)=-d_{y}(-k)$, and $d_{z}(k)=0$. The types of nonHermiticities and their manners of appearance are important for the non-Hermitian topological systems $[34,54]$. If the nonHermiticity is $\vec{\Gamma}=(0,0, \gamma)$, the topological phase transition and the existence of the edge state are unaltered because of the pseudo-anti-Hermiticity protection [34]; the topological properties of the non-Hermitian system are inherited by the EPs (exceptional rings or exceptional surfaces in $2 \mathrm{D}$ or $3 \mathrm{D}$ ) [60-67]. If the non-Hermiticity is $\vec{\Gamma}=(0, \gamma, 0)$, the nonHermitian skin effect occurs under open boundary condition [54-59,68-78], the non-Hermitian Aharonov-Bohm effect under periodical boundary condition invalidates the conventional bulk-boundary correspondence [54], and the non-Bloch band theory is developed for topological characterization [77-82]. Here, the dissipation induced anti- $\mathcal{P} \mathcal{T}$-symmetric coupling corresponds to the imaginary part $\vec{\Gamma}=(\gamma, 0,0) . h_{k}$ lacks pseudo-anti-Hermiticity protection. The inversion symmetry of $h_{k}$ ensures the absence of a non-Hermitian Aharonov-Bohm effect and the validity of conventional bulk-boundary corre- (a)

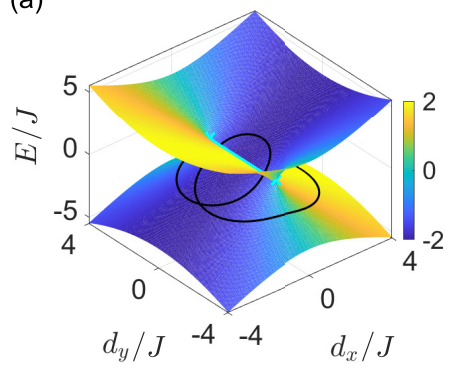

(c)

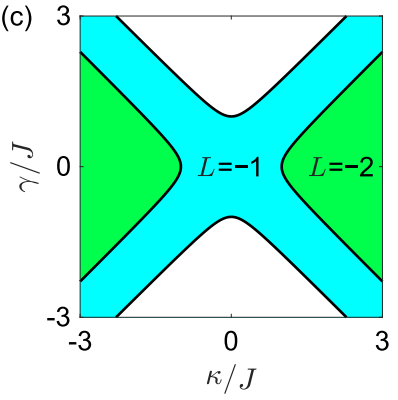

(b)

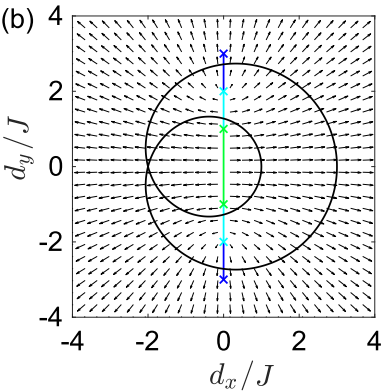

(d)

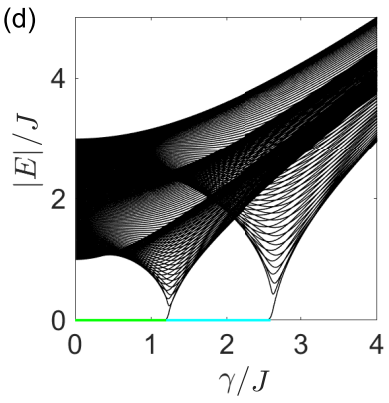

FIG. 3. (a) Complex spectrum of $h_{k}$ for $\vec{d}-i \vec{\Gamma}=\left(d_{x}-\right.$ $\left.i \gamma, d_{y}, 0\right)$ at $\gamma=2$. The color indicates the imaginary part. The solid cyan line is the bulk Fermi arc that connects two EPs (crosses). The black curve is the effective magnetic field for the photonic crystal lattice of Fig. 1 at $J=1, \kappa=2$; its projection is shown at the top of (b) $\left(\mathbf{F}_{+, x}, \mathbf{F}_{+, y}\right)$. (c) Phase diagram. (d) Energy spectrum under open boundary.

spondence. The anti- $\mathcal{P} \mathcal{T}$-symmetric coupling $\gamma$ in $h_{k}$ splits the diabolic point $\left(d_{x}=d_{y}=0\right)$ in the Hermitian case $(\gamma=0)$ into a pair of EPs [cyan crosses in Fig. 3(a)]. As $\gamma$ changes, the system switches among topologically trivial phases and different topologically nontrivial phases; therefore, the anti$\mathcal{P} \mathcal{T}$-symmetric coupling induces topological phase transition and unties the vortex link.

Figure 3(a) depicts the complex energy bands for $h_{k}=$ $\left[d_{x}(k)-i \gamma\right] \sigma_{x}+d_{y}(k) \sigma_{y}$ as functions of $d_{x}$ and $d_{y}$ when $\gamma=2$ is fixed. The Riemann surface spectrum has two EPs connected by the bulk Fermi arc, where the real part of the band gap closes. Two EPs located at $(0, \pm \gamma)$ in the $d_{x}-d_{y}$ plane are split from the diabolic point [at the origin $(0,0)$ ] for $\gamma=0$. The integer topological charge associated with the diabolic point splits into two equal half-integer topological charges [51] in the presence of anti- $\mathcal{P} \mathcal{T}$-symmetric coupling. The two-component planer vector field $\left(F_{+, x}(k), F_{+, y}(k)\right)$ is depicted in Fig. 3(b) [83]. The linking number of the vector field curves $\mathbf{F}_{ \pm}(k)$ equals the winding number of the planar vector field, $w_{ \pm}=(2 \pi)^{-1} \int_{0}^{2 \pi} \nabla_{k} \phi_{ \pm}(k) \mathrm{d} k=-L$, where $\phi_{ \pm}(k)=\arctan \left[F_{ \pm, y}(k) / F_{ \pm, x}(k)\right]$. The half-integer topological charge associated with the EP is revealed from the argument of the planar vector field $\mathbf{F}_{ \pm}(k)$. The topological charge associated with the diabolic point or EP is equal to the global Zak phase [33], which is the summation of the winding numbers of the real fictitious magnetic field loop encircling each individual EP. Notably, the global Zak phase can always be zero and invalid for topological characterization at an inappropriate gauge of the eigenmodes [84]. Thus, topological 
characterization employing the vector field also benefits from its gauge independent feature.

The topological phase transition occurs at the EPs, where the band gap closes at exact zero energy. The topological phase transition points are

$$
\gamma^{2}=\kappa^{2}+J^{2} / 2 \pm(J / 2) \sqrt{J^{2}+8 \kappa^{2}} .
$$

The phase diagram in the $\kappa-\gamma$ plane is shown in Fig. 3(c). The black curve in Fig. 3(a) indicates one energy branch of the photonic crystal lattice. The projection of the black curve in the $d_{x}-d_{y}$ plane is the real fictitious magnetic field loop $\vec{d}$ [Fig. 3(b)], which exhibits time-reversal symmetry and mirror symmetry with respect to the horizontal axis, $d_{x}(k)=d_{x}(-k)$ and $d_{y}(k)=-d_{y}(-k)$. The real fictitious magnetic field loop $\vec{d}$ passes the EPs $(0, \pm \gamma)$ at the gapless phase. In Fig. 3(b), the colored crosses indicate the EPs, and the colored lines indicate the bulk Fermi arcs at different $\gamma$. Each time the loop $\vec{d}$ comes across the bulk Fermi arc, the real part of the energy gap closes and two eigenmodes switch [85,86]. For weak non-Hermiticity, the real part of the energy bands is gapped; the fictitious magnetic field loop passes $d_{x}(k)=0$ four times as a result of the winding number $w_{ \pm}=2$. Consequently, the magnetic field loop encircles the two EPs (green crosses) twice, $F_{ \pm}(k)$ form a Solomon's knot, and the imaginary part of the energy band intersects four times [Fig. 2(a)] at fixed momenta $d_{x}(k)=0$ independent of non-Hermiticity. As nonHermiticity increases, the band gap diminishes; the vector field loop passing $\left(0, \pm d_{y}\left(k_{0}\right)\right)$ may cross the bulk Fermi arc when $|\gamma|>\left|d_{y}\left(k_{0}\right)\right|$. The crossing indicates that the band gap of the real energy closes, and the real part instead of the imaginary part of the energy band crosses at $k= \pm k_{0}$; this occurs for large non-Hermiticity. We notice two and four crossings in the real part of the energy bands in Figs. 2(c) at $\gamma=2$ and 2(e) at $\gamma=3$, respectively. In Fig. 2(c), the magnetic field loop encircles the two EPs (cyan crosses) once; in Fig. 2(e), the magnetic field loop does not encircle either of the two EPs (blue crosses).

\section{TOPOLOGICAL PHOTONIC ZERO MODES}

Topological phase transition is inextricably related to the change of topological photonic edge modes. Untying the link by one reduces one pair of edge modes. Figure 1 is a quasi-1D coupled Su-Schrieffer-Heeger chain; the termination under the open boundary condition is indicated by the red lines, the open boundary spectrum as a function of the anti- $\mathcal{P} \mathcal{T}$ symmetric coupling strength is depicted in Fig. 3(d). The edge modes are zero modes, which are analytically solvable at the limitation of infinite size, and satisfy the steady-state equations of motion

$$
\begin{aligned}
& 0=-i \gamma \psi_{A, j}+J \psi_{A, j+1}+\kappa \psi_{A, j+2}, \\
& 0=-i \gamma \psi_{B, j}+J \psi_{B, j-1}+\kappa \psi_{B, j-2} .
\end{aligned}
$$

The edge modes decay to zero from one boundary to the other. The left (right) edge modes localize within the sublattice $A(B)$ in blue (red). The steady-state equations of motion are recurrence formulas. The localized edge modes can be regarded as
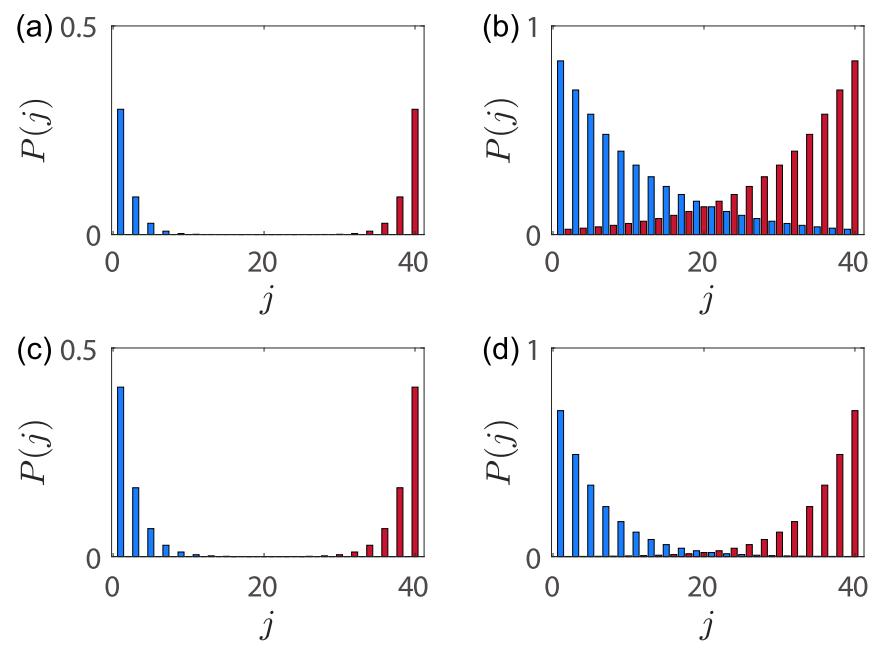

FIG. 4. Edge states in the topologically nontrivial phases. The parameters are (a) $\gamma=1$ with $\left|\rho_{+}\right|<1$, (b) $\gamma=1$ with $\left|\rho_{-}\right|<1$, (c) $\gamma=1.274$, and (d) $\gamma=2$. Other parameters are $J=1$ and $\kappa=2$. $P(j)=\left\langle\psi_{j} \mid \psi_{j}\right\rangle$. The blue (red) bar indicates the probability of the left (right) photonic edge modes on the sublattice $A(B)$.

plane waves $e^{ \pm i k j}$ with complex momenta $k$, which are zeros of the complex function

$$
f(\rho) \equiv-i \gamma+J \rho+\kappa \rho^{2}=0,
$$

where $\rho=e^{ \pm i k_{0}}=-\left(J \pm \sqrt{4 i \kappa \gamma+J^{2}}\right) /(2 \kappa)$ yields the localization length of the edge mode $\xi=\left|\operatorname{Im}\left(k_{0}\right)\right|^{-1}$ [87]. The number of edge modes at either boundary is equal to the number of zeros $f(\rho)=0$ within the region $|\rho|<1$, being identical to the linking number of the two vector field links. The gapless phase is the boundary of two gapped phases, where topological phase transition occurs. The number of topologically protected edge modes at the gapless phase is equal to that at the gapped phase with a small winding number; two (zero) topological edge modes present in the gapless phase between topological phases $L=-2(L=0)$ and $L=-1$. The left edge mode amplitude is $\psi_{A, j}=\rho^{j}$ and the right edge mode amplitude is $\psi_{B, N+1-j}=\rho^{j}$. The two pairs of edge modes at $\gamma=1$ are depicted in Figs. 4(a) and 4(b), respectively. The pair of edge modes at $\gamma=1.274$ and $\gamma=2$ are depicted in Figs. 4(c) and 4(d).

\section{CONCLUSIONS}

We investigate a dissipative quasi-1D topological photonic crystal lattice of coupled resonators. The coupled resonator array exhibits sublattice symmetry and the dissipation induced anti- $\mathcal{P} \mathcal{T}$-symmetric couplings alternatively presented. Counterintuitively, more dissipation constructively generate nontrivial topology from trivial topology. The nontrivial topology is established from the linking geometry of the vector fields, which are the average values of the Pauli matrices under the Bloch Hamiltonian. The anti- $\mathcal{P} \mathcal{T}$-symmetric coupling unties the vector field links, alters the band topology, and induces topological phase transition at the EPs. The linking number determines the number of topologically protected 
photonic zero modes. The dissipation may not affect the dissipation rate of the photonic zero modes although it may alter the band topology. Our findings deepen the understanding of the influence of dissipation induced anti- $\mathcal{P} \mathcal{T}$-symmetric coupling and the non-Hermitian 1D topology.

\section{ACKNOWLEDGMENTS}

This work was supported by National Natural Science Foundation of China (Grants No. 11975128 and No. 11874225).
[1] M. R. Dennis, R. P. King, B. Jack, K. O'Holleran, and M. J. Padgett, Isolated optical vortex knots, Nat. Phys. 6, 118 (2010).

[2] Y. Kawaguchi, M. Nitta, and M. Ueda, Knots in a Spinor BoseEinstein Condensate, Phys. Rev. Lett. 100, 180403 (2008).

[3] M. Z. Hasan and C. L. Kane, Colloquium: Topological insulators, Rev. Mod. Phys. 82, 3045 (2010); X.-L. Qi and S.-C. Zhang, Topological insulators and superconductors, ibid. 83, 1057 (2011)

[4] Y. Ashida, Z. Gong, and M. Ueda, Non-Hermitian Physics, arXiv:2006.01837.

[5] B.-Y. Xie, H.-F. Wang, X.-Y. Zhu, M.-H. Lu, Z. D. Wang, and Y.-F. Chen, Photonics meets topology, Opt. Exp. 26, 24531 (2018).

[6] T. Ozawa, H. M. Price, A. Amo, N. Goldman, M. Hafezi, L. Lu, M. C. Rechtsman, D. Schuster, J. Simon, O. Zilberberg, and I. Carusotto, Topological photonics, Rev. Mod. Phys. 91, 015006 (2019).

[7] F. Zangeneh-Nejad and R. Fleury, Topological Fano Resonances, Phys. Rev. Lett. 122, 014301 (2019).

[8] M. R. Hirsbrunner, T. M. Philip, and M. J. Gilbert, Topology and observables of the non-Hermitian Chern insulator, Phys. Rev. B 100, 081104 (2019).

[9] D.-W. Zhang, L.-Z. Tang, L.-J. Lang, H. Yan, and S.-L. Zhu, Non-Hermitian topological Anderson insulators, Sci. China Phys. Mech. Astron. 63, 267062 (2020); L.-Z. Tang, L.-F. Zhang, G.-Q. Zhang, and D.-W. Zhang, Topological Anderson insulators in two-dimensional non-Hermitian disordered systems, Phys. Rev. A 101, 063612 (2020).

[10] B. Zhu, Y. Ke, H. Zhong, and C. Lee, Dynamic winding number for exploring band topology, Phys. Rev. Research 2, 023043 (2020).

[11] H. Gao, H. Xue, Q. Wang, Z. Gu, T. Liu, J. Zhu, and B. Zhang, Observation of topological edge states induced solely by nonHermiticity in an acoustic crystal, Phys. Rev. B 101, 180303(R) (2020).

[12] X.-Q. Sun, B. Lian, and S.-C. Zhang, Double Helix Nodal Line Superconductor, Phys. Rev. Lett. 119, 147001 (2017); W. Chen, H.-Z. Lu, and J.-M. Hou, Topological semimetals with a double-helix nodal link, Phys. Rev. B 96, 041102(R) (2017).

[13] G. Chang, S.-Y. Xu, X. Zhou, S.-M. Huang, B. Singh, B. Wang, I. Belopolski, J. Yin, S. Zhang, A. Bansil, H. Lin, and M. Z. Hasan, Topological Hopf and Chain Link Semimetal States and Their Application to $\mathrm{Co}_{2} \mathrm{MnGa}$, Phys. Rev. Lett. 119, 156401 (2017).

[14] M. Ezawa, Topological semimetals carrying arbitrary Hopf numbers: Fermi surface topologies of a Hopf link, Solomon's knot, trefoil knot, and other linked nodal varieties, Phys. Rev. B 96, 041202(R) (2017).

[15] R. Bi, Z. Yan, L. Lu, and Z. Wang, Nodal-knot semimetals, Phys. Rev. B 96, 201305(R) (2017); Z. Yan, R. Bi, H. Shen, L. Lu, S.-C. Zhang, and Z. Wang, ibid. 96, 041103(R) (2017).
[16] P. J. Ackerman and I. I. Smalyukh, Diversity of Knot Solitons in Liquid Crystals Manifested by Linking of Preimages in Torons and Hopfions, Phys. Rev. X 7, 011006 (2017).

[17] J. Carlström and E. J. Bergholtz, Exceptional links and twisted Fermi ribbons in non-Hermitian systems, Phys. Rev. A 98, 042114 (2018); J. Carlström, M. Stålhammar, J. C. Budich, and E. J. Bergholtz, Knotted non-Hermitian metals, Phys. Rev. B 99, 161115(R) (2019).

[18] Z. Yang, C.-K. Chiu, C. Fang, and J. Hu, Jones Polynomial and Knot Transitions in Topological Semimetals, Phys. Rev. Lett. 124, 186402 (2020).

[19] S. Lin, L. Jin, and Z. Song, Symmetry protected topological phases characterized by isolated exceptional points, Phys. Rev. B 99, 165148 (2019).

[20] K. Wang, X. Qiu, L. Xiao, X. Zhan, Z. Bian, B. C. Sanders, W. Yi, and P. Xue, Observation of emergent momentum-time skyrmions in parity-time-symmetric non-unitary quench dynamics, Nat. Commun. 10, 2293 (2019).

[21] S. Longhi, Topological Phase Transition in Non-Hermitian Quasicrystals, Phys. Rev. Lett. 122, 237601 (2019).

[22] Q.-B. Zeng, Y.-B. Yang, and Y. Xu, Topological phases in non-Hermitian Aubry-André Harper models, Phys. Rev. B 101, 020201 (2020).

[23] F. Zangeneh-Nejad and R. Fleury, Nonlinear SecondOrder Topological Insulators, Phys. Rev. Lett. 123, 053902 (2019).

[24] X. Ni, Z. Xiao, A. B. Khanikaev, and A. Alù, Robust Multiplexing with Topolectrical Higher-Order Chern Insulators, Phys. Rev. Appl. 13, 064031 (2020).

[25] X.-W. Luo and C. Zhang, Higher-Order Topological Corner States Induced by Gain and Loss, Phys. Rev. Lett. 123, 073601 (2019); Y.-J. Wu, C.-C. Liu, and J. Hou, Wannier-type photonic higher-order topological corner states induced solely by gain and loss, Phys. Rev. A 101, 043833 (2020).

[26] T. Liu, Y.-R. Zhang, Q. Ai, Z. Gong, K. Kawabata, M. Ueda, and F. Nori, Second-Order Topological Phases in NonHermitian Systems, Phys. Rev. Lett. 122, 076801 (2019).

[27] M. Ezawa, Non-Hermitian boundary and interface states in nonreciprocal higher-order topological metals and electrical circuits, Phys. Rev. B 99, 121411(R) (2019); Non-Hermitian higher-order topological states in nonreciprocal and reciprocal systems with their electric-circuit realization, 99, 201411(R) (2019).

[28] H. Schomerus, Topologically protected midgap states in complex photonic lattices, Opt. Lett. 38, 1912 (2013).

[29] C. Poli, M. Bellec, U. Kuhl, F. Mortessagne, and H. Schomerus, Selective enhancement of topologically induced interface states in a dielectric resonator chain, Nat. Commun. 6, 6710 (2015).

[30] L. Jin, Topological phases and edge states in a non-Hermitian trimerized optical lattice, Phys. Rev. A 96, 032103 (2017). 
[31] X. Ni, D. Smirnova, A. Poddubny, D. Leykam, Y. Chong, and A. B. Khanikaev, $\mathcal{P} \mathcal{T}$ phase transitions of edge states at $\mathcal{P} \mathcal{T}$ symmetric interfaces in non-Hermitian topological insulators, Phys. Rev. B 98, 165129 (2018).

[32] S. Weimann, M. Kremer, Y. Plotnik, Y. Lumer, S. Nolte, K. G. Makris, M. Segev, M. C. Rechtsman, and A. Szameit, Topologically protected bound states in photonic parity-time-symmetric crystals, Nat. Mater. 16, 433 (2017).

[33] M. Pan, H. Zhao, P. Miao, S. Longhi, and L. Feng, Photonic zero mode in a non-Hermitian photonic lattice, Nat. Commun. 9, 1308 (2018).

[34] K. L. Zhang, H. C. Wu, L. Jin, and Z. Song, Topological phase transition independent of system non-Hermiticity, Phys. Rev. B 100, 045141 (2019).

[35] B. Wang, T. Chen, and X. Zhang, Observation of novel robust edge states in dissipative non-Hermitian quantum walks, Laser Photon. Rev. 14, 2000092 (2020).

[36] M. Hafezi, S. Mittal, J. Fan, A. Migdall, and J. M. Taylor, Imaging topological edge states in silicon photonics, Nat. Photon. 7, 1001 (2013).

[37] H. Zhao, P. Miao, M. H. Teimourpour, S. Malzard, R. ElGanainy, H. Schomerus, and L. Feng, Topological hybrid silicon microlasers, Nat. Commun. 9, 981 (2018).

[38] M. Parto, S. Wittek, H. Hodaei, G. Harari, M. A. Bandres, J. Ren, M. C. Rechtsman, M. Segev, D. N. Christodoulides, and M. Khajavikhan, Edge-Mode Lasing in 1D Topological Active Arrays, Phys. Rev. Lett. 120, 113901 (2018).

[39] P. St-Jean, V. Goblot, E. Galopin, A. Lemaitre, T. Ozawa, L. Le Gratiet, I. Sagnes, J. Bloch, and A. Amo, Lasing in topological edge states of a one-dimensional lattice, Nat. Photon 11, 651 (2017).

[40] G. Harari, M. A. Bandres, Y. Lumer, M. C. Rechtsman, Y. D. Chong, M. Khajavikhan, D. N. Christodoulides, and M. Segev, Topological insulator laser: Theory, Science 359, eaar4003 (2018).

[41] M. A. Bandres, S. Wittek, G. Harari, M. Parto, J. Ren, M. Segev, D. Christodoulides, and M. Khajavikhan, Topological insulator laser: Experiments, Science 359, eaar4005 (2018).

[42] Y. V. Kartashov and D. V. Skryabin, Two-Dimensional Topological Polariton Laser, Phys. Rev. Lett. 122, 083902 (2019).

[43] Y. Ota, K. Takata, T. Ozawa, A. Amo, Z. Jia, B. Kante, M. Notomi, Y. Arakawa, and S. Iwamoto, Active topological photonics, Nanophotonics 9, 547 (2020).

[44] A. Cerjan, S. Huang, K. P. Chen, Y. Chong, and M. C. Rechtsman, Experimental realization of a Weyl exceptional ring, Nat. Photon. 13, 623 (2019).

[45] M. Kremer, I. Petrides, E. Meyer, M. Heinrich, O. Zilberberg, and A. Szameit, A square-root topological insulator with non-quantized indices realized with photonic Aharonov-Bohm cages, Nat. Commun. 11, 907 (2020).

[46] F. Yang, Y.-C. Liu, and L. You, Anti- $\mathcal{P} \mathcal{T}$ symmetry in dissipatively coupled optical systems, Phys. Rev. A 96, 053845 (2017).

[47] J. D. Joannopoulos, S. G. Johnson, J. N. Winn, and R. D. Meade, Photonic Crystals: Modeling the Flow of Light (Princeton University Press, Princeton, NJ, 2008).

[48] T. Hofmann, T. Helbig, F. Schindler, N. Salgo, M. Brzezi ńska, M. Greiter, T. Kiessling, D. Wolf, A. Vollhardt, A. Kabaši, C. H. Lee, A. Bilušić, R. Thomale, and T. Neupert, Reciprocal skin effect and its realization in a topolectrical circuit, Phys. Rev. Research 2, 023265 (2020).
[49] X. X. Zhang and M. Franz, Non-Hermitian Exceptional Landau Quantization in Electric Circuits, Phys. Rev. Lett. 124, 046401 (2020).

[50] C. C. Adams, The Knot Book: An Elementary Introduction to the Mathematical Theory of Knots (Freeman, New York, 1994).

[51] A. A. Mailybaev, O. N. Kirillov, and A. P. Seyranian, Geometric phase around exceptional points, Phys. Rev. A 72, 014104 (2005).

[52] X. L. Zhang, S. B. Wang, B. Hou, and C. T. Chan, Dynamically Encircling Exceptional Points: In Situ Control of Encircling Loops and the Role of the Starting Point, Phys. Rev. X 8, 021066 (2018).

[53] B. Midya, H. Zhao, and L. Feng, Non-Hermitian photonics promises exceptional topology of light, Nat. Commun. 9, 2674 (2018); M. A. Miri and A. Alù, Exceptional points in optics and photonics, Science 363, eaar7709 (2019).

[54] L. Jin and Z. Song, Bulk-boundary correspondence in a nonHermitian system in one dimension with chiral inversion symmetry, Phys. Rev. B 99, 081103(R) (2019); H. C. Wu, L. Jin, and Z. Song, Inversion symmetric non-Hermitian Chern insulator, ibid. 100, 155117 (2019).

[55] Z. Gong, Y. Ashida, K. Kawabata, K. Takasan, S. Higashikawa, and M. Ueda, Topological Phases of Non-Hermitian Systems, Phys. Rev. X 8, 031079 (2018).

[56] F. K. Kunst, E. Edvardsson, J. C. Budich, and E. J. Bergholtz, Biorthogonal Bulk-Boundary Correspondence in Non-Hermitian Systems, Phys. Rev. Lett. 121, 026808 (2018).

[57] S. Yao and Z. Wang, Edge States and Topological Invariants of Non-Hermitian Systems, Phys. Rev. Lett. 121, 086803 (2018).

[58] X. Zhu, H. Wang, S. K. Gupta, H. Zhang, B. Xie, M. Lu, and Y. Chen, Photonic non-Hermitian skin effect and non-Bloch bulk-boundary correspondence, Phys. Rev. Research 2, 013280 (2020).

[59] Q.-B. Zeng, Y.-B. Yang, and R. Lü, Topological phases in one-dimensional nonreciprocal superlattices, Phys. Rev. B 101, 125418 (2020).

[60] Y. Xu, S. T. Wang, and L.-M. Duan, Weyl Exceptional Rings in a Three-Dimensional Dissipative Cold Atomic Gas, Phys. Rev. Lett. 118, 045701 (2017).

[61] H. Zhou, J. Y. Lee, S. Liu, and B. Zhen, Exceptional surfaces in $\mathcal{P} \mathcal{T}$-symmetric non-Hermitian photonic systems, Optica 6, 190 (2019).

[62] Q. Zhong, J. Ren, M. Khajavikhan, D. N. Christodoulides, S. K. Ozdemir, and R. El-Ganainy, Sensing with Exceptional Surfaces in Order to Combine Sensitivity with Robustness, Phys. Rev. Lett. 122, 153902 (2019).

[63] K. Yamamoto, M. Nakagawa, K. Adachi, K. Takasan, M. Ueda, and N. Kawakami, Theory of Non-Hermitian Fermionic Superfluidity with a Complex-Valued Interaction, Phys. Rev. Lett. 123, 123601 (2019).

[64] C. C. Wojcik, X. Q. Sun, T. Bzdušek, and S. Fan, Homotopy characterization of non-Hermitian Hamiltonians, Phys. Rev. B 101, 205417 (2020).

[65] J. C. Budich, J. Carlström, F. K. Kunst, and E. J. Bergholtz, Symmetry-protected nodal phases in non-Hermitian systems, Phys. Rev. B 99, 041406(R) (2019).

[66] R. Okugawa and T. Yokoyama, Topological exceptional surfaces in non-Hermitian systems with parity-time and parity-particle-hole symmetries, Phys. Rev. B 99, 041202(R) (2019). 
[67] T. Yoshida, R. Peters, N. Kawakami, and Y. Hatsugai, Symmetry-protected exceptional rings in two-dimensional correlated systems with chiral symmetry, Phys. Rev. B 99, 121101(R) (2019).

[68] S. Longhi, Probing non-Hermitian skin effect and non-Bloch phase transitions, Phys. Rev. Research 1, 023013 (2019).

[69] H. Jiang, L.-J. Lang, C. Yang, S.-L. Zhu, and S. Chen, Interplay of non-Hermitian skin effects and Anderson localization in nonreciprocal quasiperiodic lattices, Phys. Rev. B 100, 054301 (2019).

[70] H. Wang, J. Ruan, and H. Zhang, Non-Hermitian nodal-line semimetals with an anomalous bulk-boundary correspondence, Phys. Rev. B 99, 075130 (2019).

[71] K.-I. Imura and Y. Takane, Generalized bulk-edge correspondence for non-Hermitian topological systems, Phys. Rev. B 100, 165430 (2019).

[72] S. Longhi, Non-Bloch-Band Collapse and Chiral Zener Tunneling, Phys. Rev. Lett. 124, 066602 (2020).

[73] N. Okuma, K. Kawabata, K. Shiozaki, and M. Sato, Topological Origin of Non-Hermitian Skin Effects, Phys. Rev. Lett. 124, 086801 (2020).

[74] X. Zhang and J. Gong, Non-Hermitian Floquet topological phases: Exceptional points, coalescent edge modes, and the skin effect, Phys. Rev. B 101, 045415 (2020).

[75] X.-R. Wang, C.-X. Guo, and S.-P. Kou, Defective edge states and number-anomalous bulk-boundary correspondence in nonHermitian topological systems, Phys. Rev. B 101, 121116(R) (2020); C.-X. Guo, X.-R. Wang, C. Wang, and S.-P. Kou, Non-Hermitian dynamic strings and anomalous topological degeneracy on a non-Hermitian toric-code model with parity-time symmetry, ibid. 101, 144439 (2020).

[76] E. Lee, H. Lee, and B.-J. Yang, Many-body approach to nonHermitian physics in fermionic systems, Phys. Rev. B 101, 121109 (2020).

[77] K. Yokomizo and S. Murakami, Non-Bloch Band Theory of Non-Hermitian Systems, Phys. Rev. Lett. 123, 066404 (2019).
[78] F. Song, S. Y. Yao, and Z. Wang, Non-Hermitian Skin Effect and Chiral Damping in Open Quantum Systems, Phys. Rev. Lett. 123, 170401 (2019); Non-Hermitian topological invariants in real space, 123, 246801 (2019).

[79] K. Kawabata, K. Shiozaki, M. Ueda, and M. Sato, Symmetry and Topology in Non-Hermitian Physics, Phys. Rev. X 9, 041015 (2019); K. Kawabata, T. Bessho, and M. Sato, Classification of Exceptional Points and Non-Hermitian Topological Semimetals, Phys. Rev. Lett. 123, 066405 (2019).

[80] D. S. Borgnia, A. J. Kruchkov, and R.-J. Slager, Non-Hermitian Boundary Modes and Topology, Phys. Rev. Lett. 124, 056802 (2020).

[81] K. Kawabata, N. Okuma, and M. Sato, Non-Bloch band theory of non-Hermitian Hamiltonians in the symplectic class, Phys. Rev. B 101, 195147 (2020).

[82] T. Helbig, T. Hofmann, S. Imhof, M. Abdelghany, T. Kiessling, L. W. Molenkamp, C. H. Lee, A. Szameit, M. Greiter, and R. Thomale, Generalized bulk-boundary correspondence in nonHermitian topolectrical circuits. Nat. Phys. 16, 747 (2020); L. Xiao, T. Deng, K. Wang, G. Zhu, Z. Wang, W. Yi, and P. Xue, Non-Hermitian bulk-boundary correspondence in quantum dynamics, ibid. 16, 761 (2020).

[83] The components of the vector fields for the two bands satisfy $F_{x,+}(k)=-F_{x,-}(k), F_{y,+}(k)=-F_{y,-}(k), F_{z,+}(k)=F_{z,-}(k)$.

[84] A. I. Nesterov and F. Aceves de la Cruz, Complex magnetic monopoles, geometric phases and quantum evolution in the vicinity of diabolic and exceptional points, J. Phys. A 41, 485304 (2008).

[85] Q. Zhong, M. Khajavikhan, D. N. Christodoulides, and R. El-Ganainy, Winding around non-Hermitian singularities, Nat. Commun. 9, 4808 (2018).

[86] A. Cerjan, M. Xiao, L. Yuan, and S. Fan, Effects of nonHermitian perturbations on Weyl Hamiltonians with arbitrary topological charges, Phys. Rev. B 97, 075128 (2018).

[87] R. Verresen, N. G. Jones, and F. Pollmann, Topology and Edge Modes in Quantum Critical Chains, Phys. Rev. Lett. 120, 057001 (2018). 